\title{
Neubewertung der österreichischen Kultur der Zwischenkriegszeit
}

Verdrängte Moderne - vergessene Avantgarde. Diskurskonstellationen zwischen Literatur, Theater, Kunst und Musik in Österreich 1918-1938. Hg. Primus-Heinz Kucher. Göttingen: V\&R unipress 2016, 296 S.

\section{Wien: Vom Zentrum der Donaumonarchie zum europäischen} Avantgarde-Zentrum

Vorzustellen ist ein Band, dessen Titel etwas Investigatives verspricht, nämlich das Aufspüren verdrängter und vergessener künstlerischer Programme und Praktiken im Österreich der Zwischenkriegszeit. In diesem Sinne will der Band als Korrektiv wirken: Die Wiener Kultur der Zwischenkriegszeit, so heißt es auf dem Buchrücken, gelte als weitgehend unberührt von der sich seit 1880 etablierenden Moderne und der ihr nachfolgenden Avantgarde - eine Sicht, die der Band korrigieren will, indem er das breite Spektrum konstruktivistisch ausgerichteter Experimente an den Schnittflächen von Theater, Architektur, Literatur, Tanz und Musik rekonstruiert und dessen weit über Österreich hinaus reichende Wirkungsgeschichte verfolgt. Damit formuliert der Herausgeber, Primus-Heinz Kucher, in seinen einleitenden Bemerkungen vorsichtig die Frage, ob es denn gerechtfertigt sei, vor dem Hintergrund eher verstreuter Hinweise auf einzelne Texte, Kunstwerke, Gruppenbildungen oder Theaterexperimente die Frage nach Avantgarde und Moderne im Hinblick auf die österreichische Kunst und Kultur der 1920er Jahre überhaupt zu stellen - und gibt die Antwort, indem er Wien für die Zeit zwischen 1910 und 1930 den Status einer neben London und Berlin zu situierenden avantgardefähigen Metropole zuschreibt.

Die Vorlage für diese These bietet Hubert van den Berg, auf dessen Topographie der europäischen Avantgarde sich Kucher beruft. Entgegen der verbreiteten Annahme, dass Wien mit dem Untergang der k.u.k. Monarchie seine Anziehungskraft verloren habe, wird dann auch »eine fast ungebrochene, Projekte aufgreifende wie auch eigenständige Ideen lancierende 
kulturell-künstlerische Gemengelage « attestiert, die auch »im verkleinerten staatlichen Rahmen international ausgerichtet war« (S. 9). Die Brisanz dieser These wird deutlich, wenn man sich vor Augen führt, was unausgesprochen hinter ihr steht, die Behauptung nämlich, dass in der europäischen Welt der ersten Hälfte des 20. Jahrhunderts eine Großstadt wie Wien mehr oder weniger bruchlos künstlerisch-kulturell weiter funktionieren konnte, selbst wenn sie vom Zentrum eines bis Russland ausgreifenden Großreiches zur Hauptstadt eines Kleinstaates degradiert wurde. Und allein um Wien geht es, auch wenn im Titel des Bandes von Österreich die Rede ist. Kucher sieht in Wien »eine Art exterritoriale Enklave im zeitgenössischen Österreich» (S. 12). Dass diese Enklave zudem ein »Kreuzungspunkt verschiedener Avantgarden« (S. 12) war, wird mit Hinweisen auf avantgardistische Großereignisse belegt: die erste Präsentation russischer Konstruktivisten im Jahr 1920, eine von Kassák und Tzara 1921 organisierte Dada-Matinee, die für die internationale Wirkung des Wiener Kinetismus impulsgebende Ausstellung im Österreichischen Museum für Kunst und Industrie 1923 sowie die ein Jahr darauf unter prominenter Beteiligung und großer Resonanz abgehaltene Internationale Ausstellung neuer Theatertechnik. Zudem stützt sich Kucher auf Dieter Bogner, der für das Wien der Zeit von 1920 bis 1930 zwar eine Dominanz der traditionellen Kunstformen herausstellt, gleichwohl aber daneben (und dagegen) ein kreatives Milieu ausmacht, das theoretisch und künstlerisch an die erste Wiener Moderne anschloss und in intensivem Austausch mit der europäischen Avantgarde, insbesondere dem Konstruktivismus stand.

Auf dieses Segment der Wiener Kultur fokussiert der Band, und zwar ausdrücklich interdisziplinär, um zu »einer Neubewertung des vergessenen wie verdrängten, jedenfalls hochproduktiven literarisch-kulturell-künstlerischen Feldes der 1920er und 1930er Jahre in Österreich einen Beitrag [zu] leisten « (S. 14). Dieser Zielvorgabe folgt die Gliederung des Bandes in drei Themenblöcke: Der erste Themenblock widmet sich grundsätzlichen Überlegungen zur Wiener Avantgarde sowie dem Theater, der zweite Block setzt sich mit Entwicklungen in den Bereichen der Architektur- und Musikästhetik sowie mit den für die Moderne-Debatte maßgeblichen Zeitschriften auseinander, und der dritte Block konzentriert sich auf die Literatur im engeren Sinne.

\section{Experimentierfeld Theater und die Avantgarde als Netzwerk}

Eröffnet wird der erste Block mit dem wohl ambitioniertesten Beitrag des Bandes. Unter dem Titel »Zur Frage des >dritten Weges` in der Wiener 
Avantgarde der 1920 Jahre« erinnert der Sozialwissenschaftler Zoltan Peter zunächst an die Bedeutung des gewandelten Machtfeldes und verweist mit dem russischen Konstruktivismus und der amerikanischen Massenkultur auf die beiden neuen Pole eines Spannungsfeldes, in dem sich auch die Wiener Avantgarde verorten musste. Anschließend rekonstruiert Peter kursorisch deren Grundstruktur: Der politischen Position (Ma-Kreis) steht die unpolitische Position (u.a. Wiener Kinetismus) gegenüber. Zwischen beide Pole schiebt Peter eine dritte, laut Verfasser für die Wiener Avantgarde eigentlich charakteristische Position, der neben Karl Kraus und Béla Balázs auch der Architekt Josef Frank zugeschrieben wird. Am Beispiel des Letzteren erläutert Peter die dritte Position näher und verdichtet zur These eines Sonderwegs der Wiener Moderne; eine These, die angesichts auffallender Parallelen etwa zum Bauhaus zu prüfen wäre.

Barbara Lesák zoomt anschließend mit der österreichischen Theateravantgarde von 1918 bis 1926 an ein Subfeld des kreativen Milieus näher heran. Zweierlei soll damit erreicht werden: Zum einen sollen die durch die Politik des Austrofaschismus und in der Folge des Nationalsozialismus verfemten Bewegungen und ihre Protagonisten aus dem Dunkel der Geschichte geholt werden, zum anderen und darüber hinaus sollen diese Protagonisten typisiert werden. Drei Typen stellt Lesák nebeneinander: den elitären Typus des Visionärs oder Utopisten (Friedrich Kiesler, Rudolf Steiner oder auch Jakob Levy Moreno), der für seine Zeit zu radikal dachte; der Typus des Theoretikers (Adolf Loos und Lajos Kassák), der aus dem positiv Vorhandenen neue Wege für das Theater suchte; und der auf unmittelbare Umsetzung der Ideen zielende Typ des Theaterpraktikers bzw. der Theaterpraktikerin (Hans Fritz oder auch die Malerin Erika Giovanna Klien). Dabei liegt die Stärke des Beitrags vielleicht weniger in der Typologie als vielmehr in der Anschaulichkeit, mit der Lesák die einzelnen Protagonisten und ihre Schicksale vorstellt. Zusammengenommen bilden sie noch einmal einen ganz eigenen, für die Wiener Avantgarde insgesamt bezeichnenden Typ, den man als Weltkünstler bezeichnen könnte. So wenn Kiesler, 1890 in Czernowitz geboren, in Wien mit seiner Theaterarbeit beginnt, die Stadt jedoch 1926 verlässt, um seine Arbeit in Paris und später in New York am Universal Theatre erfolgreich fortzusetzen. Oder sein Konkurrent, der aus Rumänien stammende Moreno, 1919 in Wien eingebürgert, der Wien bereits 1925 verließ, um erfolgreich in den USA weiter zu arbeiten. Und auch die Praktikerin Klien wanderte in den 1920er Jahren in die USA aus und hinterließ Wien >nur ein - Projekt gebliebenes - >Kinetisches Marionettentheater . Insofern zeigt der Beitrag vor allem eindringlich, dass das, was unter dem, wie Lesák es nennt, »weiten Dach der österreichisch-ungarischen 
Donaumonarchie« begann, für eine Zeit in Wien zusammenkam, um von dort in andere Kunst-Zentren weiterzuziehen.

Die von Lesák nachgezeichneten Wege der Protagonisten provozieren dann auch unweigerlich die Frage, ob die Wiener Avantgarde der Zwischenkriegszeit nicht wesentlich ein Erbe der Donaumonarchie war. Die Akteure waren mehrheitlich aus der Peripherie des Reiches ins Zentrum gekommen, um dort solange zu bleiben, bis der Bedeutungsverlust Wiens nach dem Untergang des Reiches unübersehbar wurde. Dass die Avantgarde, wie es in der Einleitung des Bandes heißt, auch »im verkleinerten staatlichen Rahmen international ausgerichtet war «, erscheint nach Lektüre des Beitrags von Lesák jedenfalls eher als ein Erbe der Habsburgischen Geschichte denn als Resultat der kulturellen Anziehungskraft Wiens nach 1918. Dies geht auch aus dem nachfolgenden Beitrag von Anke Bosse hervor. Bosse konzentriert sich auf die Kooperation zwischen Karel Čapek und Friedrich Kiesler und verfolgt die von ihnen mitgetragenen Abstraktions- und Technifizierungstendenzen in der Bühnenästhetik und Bühnenpraxis bis in die Zeit um 1900 zurück. Dabei merkt man Bosses Beitrag ebenso wie dem von Lesák die langjährige Beschäftigung mit den Themenfeldern Moderne, Avantgarde, Theater an. Bei Bosse jedoch kommt etwas hinzu, mit dessen Hilfe sie das Theater als das eigentliche Experimentierfeld der Avantgarde auszuweisen vermag, nämlich eine medienwissenschaftliche Perspektive. Diese ermöglicht Bosse (Stichwort: Plurimedialität), einen sich zunehmend radikalisierenden Abstraktions- und Depersonalisierungsprozess zu rekonstruieren, an dessen Ende das Theater zur kinetischen Kunst wird und riskiert, sich selbst zu suspendieren. Die von Kiesler entworfene Vision eines optophonetischen Theaters steht eindeutig in der Tradition einer Avantgarde, die mit der Aufhebung der Kunst spielte, um es, wie wir seit Peter Bürger wissen, beim Spiel bewenden zu lassen. Das gilt, gerade der Fall Kiesler zeigt dies sehr anschaulich, auch für die Wiener Avantgarde. Gespielt wurde weiter - nur eben nicht mehr in Wien.

Komplettiert wird der Blick auf das avantgardistische Theater von Jürgen Doll, der sich mit dem sozialdemokratischen Theater jener Position zuwendet, die vor dem Hintergrund des Eröffnungsbeitrags von Peters als politische Position verstanden werden kann. Doll zeichnet die Stationen der sozialdemokratischen Theaterpraxis detailliert nach und verweist innerhalb des Subfeldes des engagierten Theaters auf eine strukturelle Differenz zwischen der Festkultur und dem revueartigen, der ProletkultBewegung nahestehenden Agitationstheater. Wie Bosse blickt Doll dabei in die Vorkriegszeit zurück und macht eine Kontinuität kenntlich, die von der Wiener Zauberposse bis zum revueartigen Agitationstheater reicht. Doch leistet Doll, liest man den Beitrag eben vor der Folie der von Peters implizit 
herangezogenen Theorie des Feldes, noch mehr: Er zeigt, wie eng der stark institutionalisierte Bereich des Theaters mit dem Feld der Politik verbunden war und wie schwierig sich gerade deshalb eine Modernisierung (jenseits visionärer Programmatik) erwies. Dies zeigt sich auch und gerade für das sozialdemokratische Theater. Mit der Niederlage der Sozialdemokratie 1934 kamen die Experimente der engagierten Position im Bereich des Theaters an ihr vorläufiges Ende.

Ganz anders der Beitrag von Arturo Larcati, mit dem der erste Block abschließt. Larcati öffnet den Fokus wieder und geht mit der Rezeptionsgeschichte des italienischen Futurismus in Wien nicht nur über den nationalen Rahmen hinaus, sondern macht die Avantgarde auch als ein gesamteuropäisches Netzwerk kenntlich, das wesentlich auf den persönlichen Kontakten einzelner Akteure und Aktivisten beruhte. Durch immer wieder neue Projekte miteinander verbunden, waren es einzelne, in ihrer Gesamtheit aber gruppenbildende Männer, die, wie Enrico Prampolini, Hans Tietze, Friedrich Kiesler und Fortunato Depero, das heterogene und in sich konkurrenzgeladene kreative Milieu zu einer relevanten Position im Feld der Kunst ausbauten und verdichteten. Von Italien über Österreich bis nach Paris und New York reichende persönliche Kontakte, Projekte und Netzwerke sind es, die Larcati hinter dem Begriff des Futurismus zum Vorschein bringt und somit die Avantgarde als das ausweist, was sie vor allem gewesen ist: eine Bewegung. Ob das Ende ihrer Glanzzeit zwingend mit dem Beginn der nationalsozialistischen Bewegung zusammenhängt, wie es Larcati nahelegt, wäre insbesondere für den Futurismus zu diskutieren. Der Beitrag selbst reißt, wie der ganz überwiegende Teil der Beiträge des Bandes, das Verhältnis von Avantgarde und Politik und damit ein ganz wesentliches Moment der Avantgardeforschung eher an, als dass er es näher untersucht.

\section{Janusköpfigkeit als Herausforderung}

Den zweiten Themenblock eröffnet der auffallend engagierte Beitrag von Evelyne Polt-Heinzl über Oskar Strnad, einen laut Verfasserin unterschätzten Pionier der Moderne. Nun gibt es derer viele, weshalb jede Wiederentdeckung wohl begründet sein will. Polt-Heinzl gelingt dies, indem sie das Profil eines Architekten nachzeichnet, der jenseits manifester Programmatik neue Akzente setzte, und zwar sowohl durch seinen antiautoritären und kooperativen Arbeitsstil wie auch durch die praktische Anwendung und Weiterentwicklung der Leitkonzepte der Moderne. Strnad wird bei Polt-Heinzl zum Anstoß für ein Überdenken der Architekturgeschichtsschreibung. In 
dieser Geschichte dominieren Akteure wie Loos, die für sich den Typus des modernen Künstlers reklamierten, während Strnad, sich mit konkreten Fragen sozial verantwortlichen Gestaltens und Bauens beschäftigend, zur Randfigur wird, und dies, obwohl er, wie der Beitrag überzeugend zu zeigen vermag, mit seinen Raum- und Beleuchtungslösungen für die Bühne weit in die Theatergeschichte hinein wirkte.

Dem klar strukturierten und informativen Beitrag von Polt-Heinzl folgt ein mehr als 30 Seiten langer Artikel mit dem nicht weniger voluminösen Titel »Ernst Krenek, Theodor Wiesengrund-Adorno und der >Begriff der Avantgarde, mit dem man in Deutschland heute nicht gern zu tun hat . Dissonanzen zu: Fortschritt und Reaktion, (alt-)neuen Formen und dem Phänomen Surrealismus«. Länge und Titel des Beitrags zeigen an, was die Verfasserin, Rebecca Unterberger, will, sehr viel nämlich, und dies im Aufsatz-Format. Dabei wird die Fülle von Zitaten weder entlang einer These noch eines ersichtlichen roten Fadens ausgebreitet, was umso mehr zu bedauern ist, als sich gerade an Kreneks Position der katholischen Avantgarde die Ambivalenz der Moderne als einer auf das Primat der Form setzenden Konzeption hätte aufzeigen lassen. Unterberger tut dies nicht. Zwar finden sich einige der in diesem Zusammenhang zentralen Begriffe wie konservative Revolution oder europäische Intelligenz, doch gehen sie im Dickicht von Zitatfetzen unter.

Wer in dem Band (auch) Einsichten in die Ambivalenz der Moderne sucht, wird in dem Beitrag von Julia Bertschik fündig. Unter dem Titel »Janusköpfige Moderne « geht Bertschik der Gradwanderung der Berliner Zeitschrift »Der Querschnitt« zwischen künstlerischer Avantgarde, Neuer Sachlichkeit und `Habsburgischem Mythos` nach. So findet sich etwa, wie die Autorin zeigt, gleich im ersten Heft des seit 1929 vom Österreicher Victor Wittner verantworteten »Querschnitt« der Artikel »Die hundert Familien. Österreichische Gesellschaft gestern und heute«, verfasst von Karl Tschuppik, der die österreichische Aristokratie zu einem »Kunstwerk aus Fleisch und Blut« erklärt, das den "politischen Bau« - gemeint ist der »Untergang Österreichs und die Degradierung Wiens zur Hauptstadt eines kleines Landes« - überlebt habe. In scheinbar krassem Gegensatz dazu steht der insgesamt eher neusachliche Diskurs der Zeitschrift; ein Gegensatz, der jedoch laut Bertschik als heterogenes Konzept vom Ullstein-Verlag geradezu zu einer »Diskurs- und Marketingstrategie« entwickelt wurde. Auf ein möglichst breites Publikum gerichtet, kombinierte die Ullstein-Kultur ganz widersprüchliche Interessen und Genres $\mathrm{zu}$ einem eigenen Raum kommunikativer Interaktion. Aus diesem Grund, und dies ist für Bertschik der entscheidende Punkt, sind Neue Sachlichkeit und Habsburgischer Mythos nicht, wie es sich seit Magris durchgesetzt hat, 
als unvereinbare Gegensätze zu sehen, sondern als eine spezifisch moderne, hybride Mischung, wie sie sich auch und insbesondere in der journalistischen Gebrauchsästhetik von Joseph Roth findet.

Dass sich die von Bertschik klar herausgestellte Janusköpfigkeit der Moderne nicht nur in Zeitgeistmagazinen sondern auch in Fachzeitschriften der Zwischenkriegszeit findet, belegt der sich anschließende Beitrag des Herausgebers Primus-Heinz Kucher. Mit den »Musikblätter[n] des Anbruch « widmet sich Kucher der zentralen publizistischen Plattform moderner Musik; eine Plattform, die, und allein dies zeigt die Widersprüchlichkeit der Avantgarde, zugleich das Hausorgan des den Markt dominierenden Musikverlags Universal Edition war. Um die Position der "Musikblätter « im internationalen Feld der Avantgarde zu bestimmen, wird eine vergleichende Diskursanalyse unternommen, mit dem Ergebnis, dass sich die Position der "Blätter" einer einfachen Bestimmung entzieht, ja sie im Grunde keine eigene Position vertraten, und doch als Plattform gelten können, die wesentliche Anliegen der Avantgarde im Bereich der Musik zur Diskussion stellte und einer breiten Rezeption zuführte. Dabei zeigen sich deutliche Parallelen zu der von Bertschik für die Moderne als symptomatisch herausgestellten Diskursstrategie: Kucher spricht von einer Heterogenisierung des Spektrums an Autoren und Perspektiven und einer »offenbar (kultur- und musikpolitisch) gefilterten Strategie«.

Dass sich die Vielfalt der Moderne-Konzeptionen im Bereich der Musik nicht auf einen gemeinsamen Nenner bringen lässt, wird von Kucher zurecht als »interpretative Herausforderung " gewertet. Die auf Dauer gestellte Vielfalt begrifflich-methodologisch einzufangen bzw. das theoretisch zu erfassen, was mit Bertschik als Ullstein-Kultur bezeichnet werden kann, bereitet den Kulturwissenschaften (noch immer) Probleme. Um der Komplexität der Verhältnisse gerecht zu werden, bedarf es eines theoretischen Rüstzeugs, das auch in den Beiträgen des hier zu besprechenden Bandes, wenn überhaupt, so eher versteckt zum Einsatz gebracht wird. Mehr Mut zur Theorie hätte dem Band gut getan, zumal es nach langen Jahren nicht nur der Avantgardeforschung sondern auch der Theorieentwicklung keinen Grund zur Zurückhaltung gibt, nachgerade wenn man über die Aufdeckung vergessener Akteure und Diskurse zu neuen Einsichten bezüglich der Moderne gelangen möchte.

\section{Themenblock Literatur}

Die Theoriescheue des Bandes gilt weitestgehend auch für den dritten und letzten Themenblock. Er widmet sich der Literatur und wird von Walter 
Fähnders mit einem Beitrag zum Zusammenspiel von avantgardistischer Lebensform und Vagabondage eröffnet, der Kennern der AvantgardeThematik in Teilen bekannt vorkommen mag. Nichtsdestoweniger ist Fähnders Beitrag erhellend, unterstreicht er doch, wie sehr die Idee der Avantgarde, also die Vorstellung einer neuen Kultur und Gesellschaft, durch eine Lebenspraxis umgesetzt wurde, mit der sich die Akteure politisch exponierten. Zumindest für die von Fähnders in Erinnerung gerufenen Vertreter der Vagabondage (Hugo Sonnenschein, Emil Szittya und Lajos Kassák) blieb es hinsichtlich der Außenseiter-Rolle nicht bei der Rhetorik. Mit dem Fokus einerseits auf der Avantgarde und andererseits auf der Heimatlosigkeit als Referenzpunkt des Lebens und Schreibens rücken hier nicht nur zwei spezifisch moderne Aspekte in den Vordergrund, sondern es wird auch ein konkreter, für die Moderneforschung relevanter Nachweis erbracht, dass nämlich zwischen der Avantgarde als Lebensform und der ästhetischen Avantgarde Berührungspunkte zwar möglich aber nicht zwingend sind, wobei jedoch nur im Fall ihrer Vermittlung als einem Akt der Selbstermächtigung ein neuer Mensch konstruiert wird. Der von Fähnders gewagte vergleichende Blick auf die Ebene des Textes und der Lebensform steht beispielhaft für eine Avantgardeforschung, die, entlang ihrem Gegenstand, immer schon die engen Grenzen der Literaturwissenschaft übertrat.

Der Beitrag von Vivien Boxberger zur Emanzipation der >Neuen Tochter in Mela Hartwigs Das Verbrechen folgt dieser Bewegung nicht und verfährt ganz im Modus des >close reading ‘. Zwar liegt der von Boxberger interpretierte Roman aus dem Jahr 1927 im zeitlichen Fokus des Bandes, ob er allerdings in einem thematischen Bezug zu Avantgarde und Moderne steht, mag man nach der Lektüre des Beitrags bezweifeln. Die auf der Textebene stattfindende avantgardistische Kunstzerstörung zumindest wird eher behauptet als nachgewiesen. Als ungleich aufschlussreicher erweist sich die nachfolgende Fallstudie von Jürgen Egyptien zur Dramatik von Ernst Fischer. Egyptien versteht es, die Entwicklung von Fischer so zu kontextualisieren, dass in ihr symptomatische Züge der widersprüchlichen Kulturgeschichte der Moderne sichtbar werden: Angefangen vom Lebensstil der Bohème über die Politisierung und Modernisierung der ästhetischen Verfahren bis hin zur formkonservativen Kehrtwende, wie sie Fischer nach den Ereignissen vom 15. Juli 1927 mit einem Genre vollzog, das Egyptien treffend als »Konversationsstücke zum Thema Revolution «bezeichnet. Dabei unterstreicht der Fall Fischer noch einmal zwei wesentliche Momente der Moderne: Zum einen das Ringen mit der Form, d.h. die Schwierigkeit, den in literaturkritischen Schriften geäußerten Willen zu neuen Formen (hier der Dramatik) in der Praxis umzusetzen und, zum zweiten, das Ausgreifen 
der Formfrage und mithin eines genuin ästhetischen Moments in das Politische. In seiner neusachlichen Phase, so Egyptien, sah Fischer Kunst und Politik als Laboratorium an und markiert somit eine Position, auf welcher die kulturwissenschaftlich faszinierende Dynamik der Zwischenkriegszeit gründet.

Gänzlich in den Mittelpunkt gerückt wird die Position der Neuen Sachlichkeit einschließlich des ihr innewohnenden entgrenzenden Potentials von Aneta Jachimowicz. Ihr Beitrag zum Romanprojekt Karl und das 20. Jahrhundert von Rudolf Brunngraber schließt den Band gebührend ab, erinnert er mit dem Projekt doch eindringlich daran, wie eng verwoben Kunst, Wissenschaft und Politik in der Zwischenkriegszeit waren. Jachimowicz konzentriert sich wesentlich auf die ideengeschichtliche Grundlage und rekonstruiert die Beziehung zwischen dem austromarxistischen Theoretiker und Mitbegründer der Sozialstatistik Otto Neurath und dem Schriftsteller Brunngraber. Beiden, so will Jachimowicz zeigen, ging es um Aufklärung für die Massen. Dieser Funktionszuschreibung versuchte der sozialdemokratische Wiener Schriftsteller mit einem rational-statistischen Erzählgestus formal zu entsprechen, d.h. mit der Einbindung des statistischen Diskurses in den Roman. Mit diesem Verfahren wiederum wollte Brunngraber nicht weniger, als die neue literarische Gattung des soziologischen Romans begründen. Über Jachimowicz hinaus kann diese Gattung, ganz wie der erkenntnistheoretische Roman eines Musil oder Broch, als das genuine Produkt einer Krisenerfahrung verstanden werden, in der die Krise der Gesellschaft, der Kultur, des Ichs und der Wirklichkeitswahrnehmung zur Krise des Erzählens wurde und die in der Tat neue literarische Formen hervorbrachte. Dass diesen zumindest im Fall Brunngraber keine nachhaltige Wirkungsgeschichte vergönnt war, ändert nichts daran, dass, wie der Beitrag deutlich macht, der Gesellschaftsroman mit dem soziologischen Roman Brunngrabers eine neue Reflexionsstufe erreichte.

\section{Fazit oder: Ist das Ganze mehr als die Summe seiner Teile?}

Womöglich, dies hätte eine Geschichte des Gesellschaftsromans im 20. Jahrhundert zu klären, markiert der von Jachimowicz wiederentdeckte soziologische Roman den vorläufigen Endpunkt der Genese einer Subgattung - nur eine der zahlreichen im Band aufgedeckten transdisziplinären Spuren, denen nachzugehen sich lohnte. Denn in diesem Punkt hält der Band, was er verspricht: Er ist interdisziplinär und leistet zweifelsfrei einen Beitrag zur Neubewertung des »vergessenen wie verdrängten, jedenfalls 
hochproduktiven literarisch-kulturell-künstlerischen Feldes der 1920er und 1930er Jahre in Österreich «. Dies allerdings mit drei Einschränkungen, denn erstens handelt es sich, wie gesagt, nicht um Österreich sondern allein um Wien. Zweitens, und zu dieser Überzeugung kommt man nach der Lektüre einmal mehr, handelt es sich bei der vergessenen Avantgarde womöglich weniger um ein Feld als vielmehr um eine hochproduktive, mit dem Traditionalismus konkurrierende Position im Spannungsfeld der Moderne. $\mathrm{Zu}$ verstehen wären die avantgardistische Position wie auch ihr Vergessen dann aber nur im theoretisch erweiterten Rahmen eines Feldes widerstreitender Positionen, in dem sich die Avantgarde (allein) nicht durchzusetzen vermochte. Vielleicht wäre bei einer solchen, behutsam theoriegeleiteten Erweiterung, und dies ist die dritte Einschränkung, etwas noch stärker ins Blickfeld geraten, das die leitende These einer in der Zwischenkriegszeit zwar überaus aktiven, von der nachfolgenden Kulturgeschichte aber verdrängten Moderne erheblich relativiert hätte, die Tatsache nämlich, dass namhafte Akteure in den 1920er Jahren Österreich bereits verließen und ihre Arbeit in anderen Avantgarde-Zentren fortsetzten - bis sie auch in und aus ihnen verdrängt wurden.

Doch sind die Schwächen des Bandes gleichsam seine Stärke. Sie sind der Intention geschuldet, Wien auch nach 1918 auf der Karte der Avantgarde-Zentren zu halten und erweisen sich in dieser Hinsicht als überaus produktiv. Das von den Beiträgen aufgerissene Spektrum konstruktivistisch ausgerichteter Experimente ist, ganz wie der Buchrücken verspricht, bemerkenswert und durchaus dazu angetan, neue Akzente zum Epochenprofil der Zwischenkriegszeit zu setzen. Mehr noch als die zentrale Rolle Wiens für das europäische Netzwerk der Avantgarde führt der Band vor Augen, dass die Würfel im Wien der 1920er Jahre noch nicht gefallen waren. Gerade in diesem Sinne ist nicht nur jeder Beitrag für sich investigativ, vielmehr erweisen sich die Beiträge in der Summe als überaus instruktiv: Zusammengenommen decken sie auf, was an kulturellen Tendenzen vorhanden bzw. im Möglichkeitsraum der europäischen Kulturgeschichte vorrätig war und provozieren einmal mehr die Frage, warum sich in der retrospektiv entscheidenden Epoche des 20. Jahrhunderts die spezifisch moderne kulturell-künstlerische Gemengelage nicht halten konnte und einer Position weichen musste, die jeder widersprüchlichen Situation ein Ende zu bereiten versprach. Eine Frage von anhaltender Relevanz, die, ganz wie der Band es vormacht, nur transdisziplinär beantwortet werden kann. 